\title{
A Comparative Study on the Effects of Domperidone, Metoclopramide, Clebopride and Trimebutine on the Gastro-Duodenal Preparation of the Guinea Pig
}

\author{
Jan A.J. SCHUURKES, Ludo F.M. HELSEN and Jan M. VAN NUETEN \\ Department of Pharmacodynamics, Janssen Pharmaceutica Research Laboratories, \\ B-2340 Beerse, Belgium
}

Accepted June 3. 1985

\begin{abstract}
Domperidone (dopamine antagonist), metoclopramide and clebopride (both dopamine antagonists and stimulators of the intramural cholinergic system). and trimebutine (spasmolytic) are used in the treatment of digestive disorders such as dyspepsia or gastritis. Our aim was to compare the effects of these compounds on the isolated intact gastroduodenal preparation of the guinea pig. Domperidone $\left(1 \mathrm{C} 50=10^{-6} \mathrm{M}\right)$, clebopride $\left(10^{-5} \mathrm{M}\right)$ and metoclopramide $\left(2 \times 10^{-5} \mathrm{M}\right)$ antagonized gastric relaxations induced by dopamine. In contrast with clebopride, domperidone and metoclopramide enhanced the amplitude of gastric contractions, moderately reduced contractile frequency, and enhanced antroduodenal coordination in a dosedependent manner ( $E$ C50 for domperidone $3 \times 10^{-7} \mathrm{M}$, for metoclopramide $2 \times 10^{-5}$ M). Trimebutine reduced gastric spontaneous activity and antroduodenal coordination. Trimebutine had a direct relaxatory effect on gastric tone (EC50= $\left.4 \times 10^{-6} \mathrm{M}\right)$. The mechanism of this inhibitory effect remains unknown but our data indicate that it is not mediated via dopamine or opiate receptor subtypes. Conclusion: domperidone, clebopride, metoclopramide and trimebutine exert distinct and diverse effects on the motility parameters of the gastroduodenal preparation of the guinea pig. These diverging actions may help explain the differences in patients' responsiveness to the treatment of digestive disorders such as dyspepsia or gastritis.
\end{abstract}

Domperidone is widely used for upper gastrointestinal discomfort, varying from nausea and vomiting to milder dyspeptic symptoms (1). Pharmacologically, domperidone is a selective dopamine antagonist (2. 3). To elucidate the mechanism of action of prokinetic compounds like domperidone. the gastroduodenal preparation of the guinea pig has been studied (2). In this preparation. domperidone was shown to enhance antroduodenal coordination via specific dopamine receptors located on intramural nerves (4) Other prokinetic dopamine antagonists like metoclopramide and clebopride have, in addition to their dopamine antagonistic properties, direct stimulatory effects on the gastrointestinal cholinergic innervation most likely by enhancing the release of acetyl- choline (5, 6). Recently, the spasmolytic trimebutine, a compound not interfering with either the cholinergic or dopaminergic system. was tested in dyspeptic patients (7).

The aim of the present study was to compare the motility effects of domperidone, metoclopramide, clebopride and trimebutine on the gastroduodenal preparation of the guinea pig

\section{Materials and Methods}

Pirbright guinea pigs (350-450 g. fasted overnight) of either sex were killed by cervical dislocation. The stomach and the first $10 \mathrm{~cm}$ of the duodenum with adhering vasculature were removed for experimentation. The intraluminal contents were removed by repeated washing. 
After ligation of the esophagus. the stomach was filled with $20 \mathrm{ml}$ saline and suspended in $200 \mathrm{ml}$ of oxygenated $\left(95 \% \mathrm{O}_{2}\right.$. $5 \% \mathrm{CO}_{2}$ ) Krebs-Henseleit solution $(\mathrm{KCl}, 4.7$ : $\mathrm{CaCl}_{2} \cdot 2 \mathrm{H}_{2} \mathrm{O}$. 2.5; $\mathrm{MgSO}_{4} \cdot 7 \mathrm{H}_{2} \mathrm{O}, \quad 1.2$; $\mathrm{NaHCO}_{3}, 25.0 ; \mathrm{KH}_{2} \mathrm{PO}_{4}, 1.2 ; \mathrm{NaCl}, 118.1$; glucose, $5.55 \mathrm{mM}$ ). maintained at $37^{\circ} \mathrm{C}$. The duodenum was cannulated and connected with an ultrasonic transit time transducer (Janssen Scientific Instruments Division) to record changes in intraluminal volume and with a bottle of saline to ensure a constant hydrostatic pressure of $6 \mathrm{~cm} \mathrm{H} \mathrm{H}_{2} \mathrm{O}(2,8)$.

Gastric relaxation, as quantified by incremental changes in gastric volume, was induced pharmacologically by intra-arterial injection of dopamine (Janssen Chimica) $\left(2.6 \times 10^{-7}\right.$ moles) via the left gastric artery in volumes of $50 \mu \mathrm{\mu l}$ at intervals of $10 \mathrm{~min}$. When reproducible relaxations were obtained, a compound was administered to the bathing solution. Drug effects were followed for 70 min. To correct for the gradual increase in agonist-induced relaxation with time in the control experiments, the control responses (expressed as \% of their initial relaxation at $t_{0}$ ) were taken as $100 \%$ at each time point: $t_{0}$. $t_{10}, t_{20} . . . t_{70}$. Agonist-induced relaxations in the presence of a compound (in \% of initial value) were then individually calculated as $\%$ of the value at the corresponding time point in the control experiments. Doseresponse curves were made by plotting the percentage inhibition of agonist-induced relaxation against the concentration of a compound.

Spontaneous phasic activity was always present on the stomach and recorded as rhythmic changes in gastric volume. Gastric peristaltic waves either stopped at the pylorus or were propagated to the duodenum (4). The passage of the antral wave to the duodenum was marked on the recording paper. Antroduodenal coordination was quantified as the relative number of antral waves that were propagated to the duodenum, i.e., that were followed by a duodenal contraction in the second quarter of the rhythmic pen deflection. Autonomous duodenal contractions (not linked to the antral wave) were observed only exceptionally and therefore not taken into account. After a 30-min stabilization period. domperidone (Janssen), clebopride (Almirall) metoclopramide (Delagrange) or trimebutine (Jouveinal) were added to the bath solution at varying concentrations. Drug effects were followed for $30 \mathrm{~min}$. The effects of trimebutine were also assessed in the presence of domperidone $\left(6 \times 10^{-6} \mathrm{M}\right)$ or naloxone (Endo) $\left(4.5 \times 10^{-7} \mathrm{M}\right)$. Three parameters were determined: 1) antral contractile amplitude, expressed as $\mathrm{ml}$ of expelled volume; 2) antral frequency, measured as number of contractions per minute; and 3 ) $\%$ antroduodenal coordination. Results were statistically evaluated with Student's twotailed $t$-test for paired or unpaired observations $(P<0.05)$.

\section{Results}

Intra-arterial injection of dopamine induced reproducible relaxations of the gastroduodenal preparation (Fig. 1). These relaxations could be inhibited upon administration of domperidone in the bathing solution. The maximal effect of domperidone was reached after $50 \mathrm{~min}$ (Fig. 1). Interestingly, as demonstrated in Fig. 1, domperidone also regularized the spontaneous motor activity and enhanced its amplitude already within $10 \mathrm{~min}$ after its administration. Clebopride (Fig. 1) and metoclopramide also effectively antagonized the dopamine-induced relaxations. In contrast with domperidone, their effects peaked at $10 \mathrm{~min}$.

The direct effects of the test compounds on spontaneous contractile activity and tone are better demonstrated at higher paper speed (Fig. 2). Domperidone enhanced the amplitude of the antral contractions and induced duodenal contractions to occur at the same frequency as the antral waves, i.e.. domperidone induced a coordinated pattern of antroduodenal motility (Fig. 2). Similar findings were obtained with metoclopramide but only in some $\left(4 / 6\right.$ at $\left.1.2 \times 10^{-6} \mathrm{M}\right)$ experiments with clebopride. In contrast to the latter drugs, trimebutine (Fig. 2) reduced both the tone of the preparation and the amplitude of the spontaneous gastric contractions. In preparations with duodenal activity, trimebutine nhibited the occurrence of these contractions. The gastric relaxation induced by trimebutine persisted throughout 
A
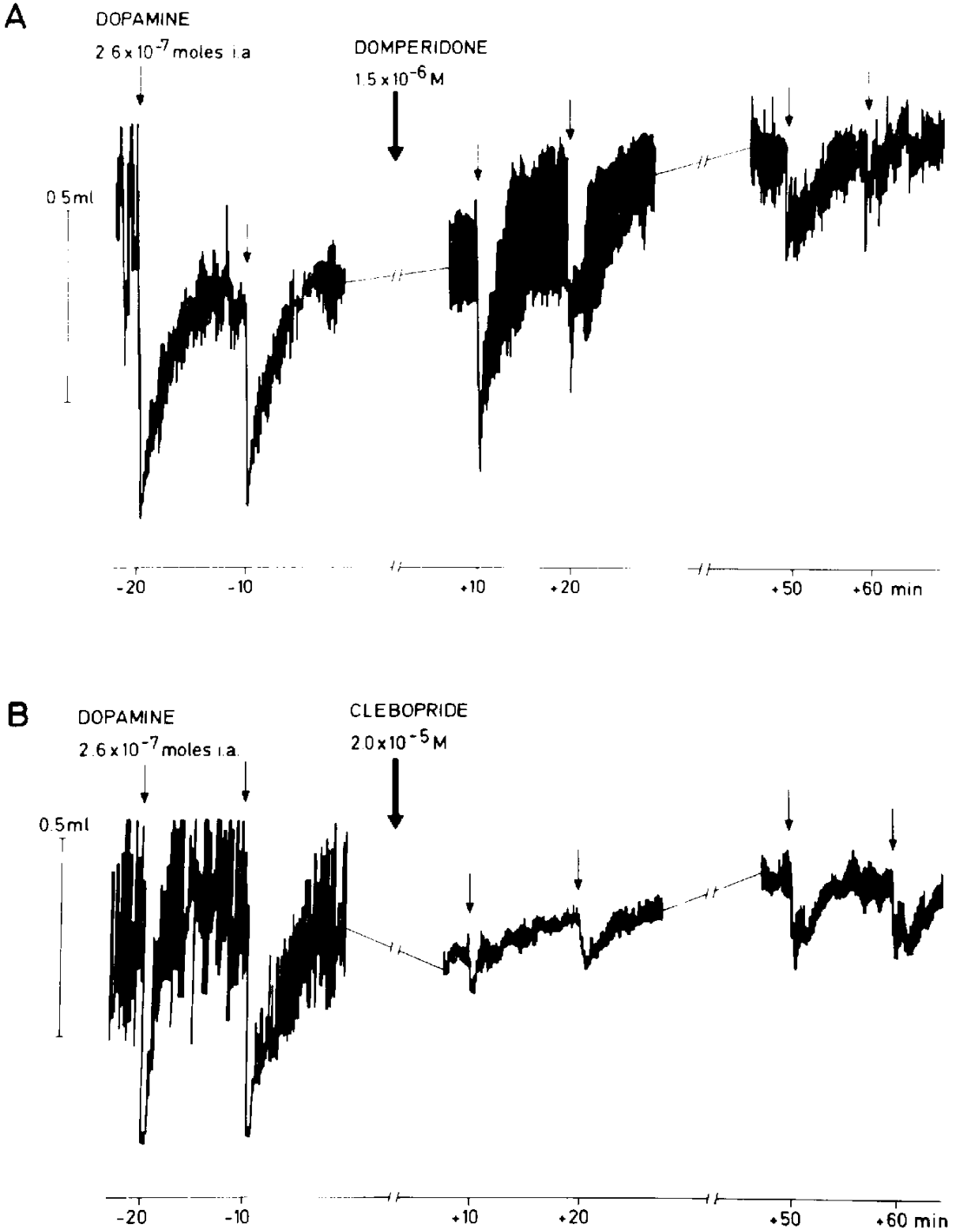

Fig. 1. Antagonism by domperidone (A) and clebopride (B) of relaxations of the gastroduodenal preparation of the guinea pig. induced by intra-arterial (i.a.) injection of dopamine (every 10 min).

the experiment (Fig. 2). It could not be prevented by domperidone $\left(6 \times 10^{-6} \mathrm{M}\right)$ nor reversed by naloxone $\left(4.5 \times 10^{-7} \mathrm{M}\right)$. This effect was dose-dependent (Fig. 3) with an EC50-value of $3.6 \times 10^{-6} \mathrm{M}$ [lower limit $(L L)=2.4 \times 10^{-6} \mathrm{M}$; upper limit $(U L)=5.5 \times$ $\left.10^{-6} \mathrm{M}\right]$. Due to these intrinsic relaxatory effects of trimebutine, its effect on dopamineinduced relaxation could not be tested.

Dose-response studies revealed that domperidone was most effective in antagonizing gastric relaxations induced by dopamine (Fig. 4). Its IC50-value was $1.0 \times 10^{-6} \mathrm{M}\left(\mathrm{LL}=0.6 \times 10^{-6} \mathrm{M}, \mathrm{UL}=1.6 \times 10^{-6}\right.$ 
A

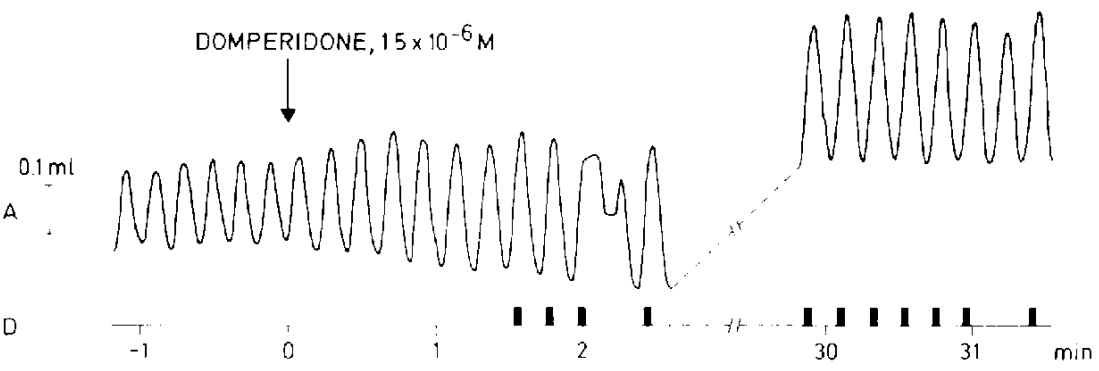

B

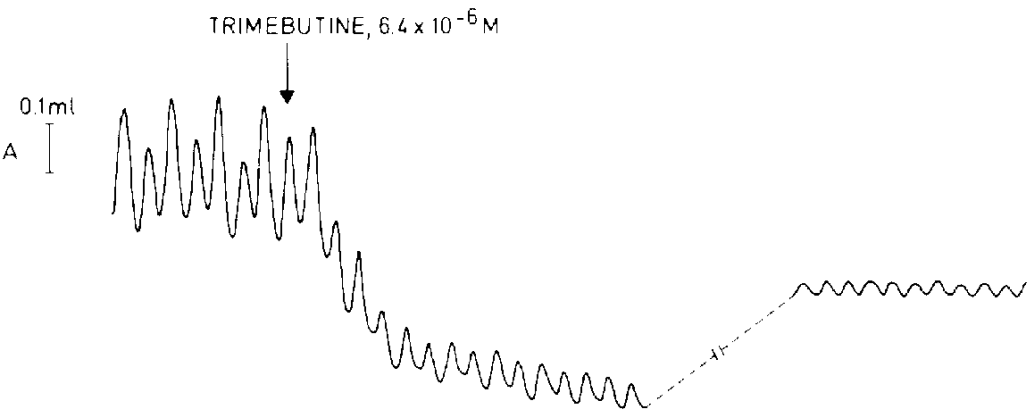

D

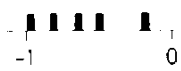

1

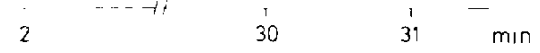

Fig. 2. Effects of domperidone (A) and trimbutine (B) on spontaneous rhythmic activity and basal tone in the gastroduodenal preparation of the gumea pig. A=antral contractile activity, $D=$ occurrence of a coordinated duodenal contraction.

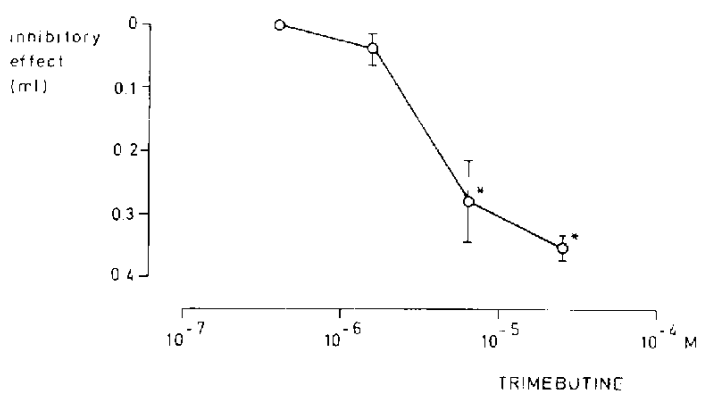

Fig. 3. Dose-response relation of the inhibitory effect (in $\mathrm{ml}$ ) of trimebutine on gastric tone (i.e. relaxation) in the guinea pig gastroduodenal preparation ( $\bar{m} \pm$ S.E.M., $n=5$, "significant decrease in tone. $P<0.05)$.

M). In this regard, domperidone was 10 and 17 times more potent than clebopride and metoclopramide, respectively. The $1 \mathrm{C50}-$ value for clebopride was $1.0 \times 10^{-5} \mathrm{M}$ $\left(\mathrm{LL}=5.7 \times 10^{-6} \mathrm{M}, \mathrm{UL}=1.7 \times 10^{-5} \mathrm{M}\right)$; for metoclopramide $1.7 \times 10^{-5} \mathrm{M}\left(\mathrm{LL}=1.0 \times 10^{-5}\right.$ M. UL=3.0 $\times 10 \mathrm{M}^{-5}$ ).
Both domperidone and metoclopramide significantly enhanced gastric contractile amplitude (Fig. 5), but their effects were only observed at a single concentration. The effective concentration of domperidone appeared 34 times lower than that needed for metoclopramide. Clebopride did not enhance the amplitude significantly. Trimebutine reduced contractile amplitude (Figs. 1 and 5). At the optimal concentrations tested, only domperidone and metoclopramide had significant effects, i.e., reduced the frequency of the gastric contractions (Fig. 6).

Both domperidone (Fig. 2) and metoclopramide enhanced antroduodenal coordination in a dose-dependent manner (Fig. 7). The EC50-value for domperidone was $2.8 \times 10^{-7} \mathrm{M}\left(\mathrm{LL}=1.6 \times 10^{-7} \mathrm{M}, \mathrm{UL}=4.7 \times 10^{-7}\right.$ $M)$. In this regard, domperidone was 160 times more potent than metoclopramide, its EC50-value being $2.2 \times 10^{-5} \mathrm{M}\left(L \mathrm{~L}=1.7 \times 10^{-5}\right.$ $\left.\mathrm{M}, \mathrm{UL}=2.7 \times 10^{-5} \mathrm{M}\right)$. Clebopride moderately enhanced antroduodenal coordination (Fig. 7), but only at $1.2 \times 10^{-6} \mathrm{M}$. From this 


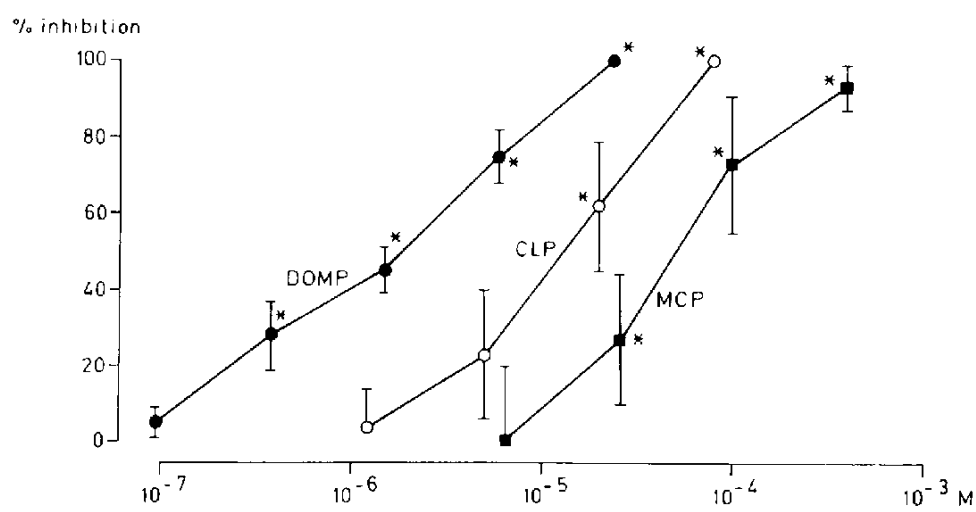

Fig. 4. Dose-response relation of the inhibitory effects of domperidone (DOMP. O), clebopride (CLP. $O$ ) and metoclopramide (MCP. $\square$ ) on dopamine-induced gastric relaxations of the guinea pig gastroduodenal preparation ( $m \pm$ S.E.M., $n=7$, " significantly different from the solvent value, $P<0.05$ ).
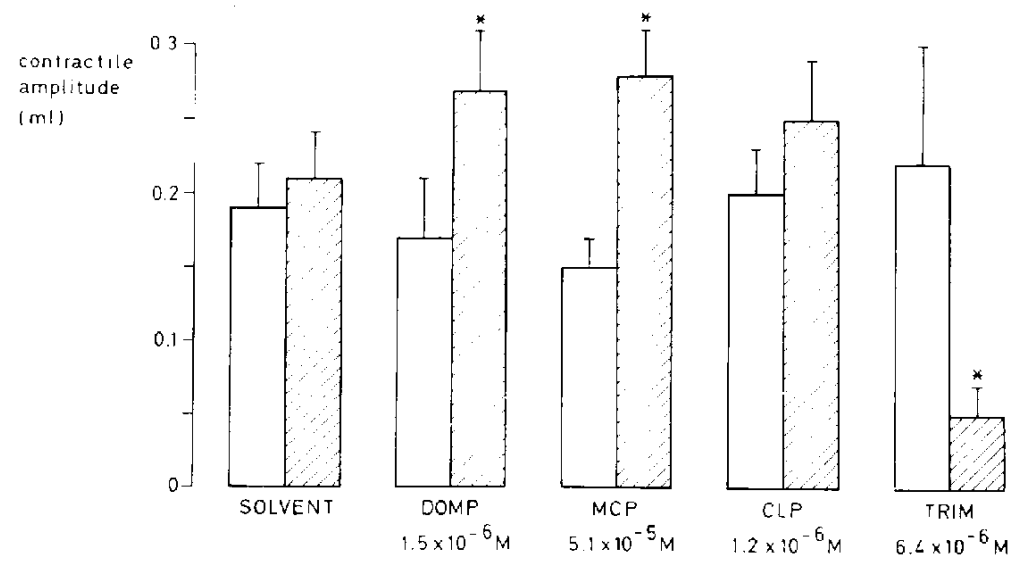

Fig. 5. Effects of domperidone (DOMP). metoclopramide (MCP), clebopride (CLP) and trimebutine (TRIM) at optimal concentrations on antral contractile amplitude [expressed in volume changes ( $\mathrm{ml}$ )] of the guinea pig stomach (open bars: initial values, hatched bars: values in the presence of the test compound, $\bar{m} \pm$ S.E.M. $n=5-9$, * significantly different from the initial value, $P<0.05)$.

concentration upwards, the compound induced in some experiments $(3 / 7$ at $4.9 \times 10^{-6} \mathrm{M}: 5 / 6$ at $\left.2.0 \times 10^{-5} \mathrm{M}\right)$ a shortlasting gastric relaxation and inhibition of contractile amplitude. Trimebutine significantly reduced antroduodenal $\mathrm{CO}-$ ordination (Fig. 7). The EC50-value for this effect of trimebutine was $5.5 \times 10^{-6} \mathrm{M}$ $\left(L L=3.1 \times 10^{-6} \mathrm{M}, \quad U L=9.7 \times 10^{-6} \mathrm{M}\right)$.

\section{Discussion}

These results clearly indicate that the four test compounds exerted different effects on the gastroduodenal preparation of the guinea pig.

Domperidone antagonized the relaxatory effects of dopamine at low concentrations $(2,8)$. In this respect, domperidone was more potent than either clebopride or metoclopramide. The potency order of these compounds is similar to that found for their respective binding affinity to striatal dopamine receptors in vitro $(3,9)$ as well as for their ED50-values as antagonist against apomorphine-induced vomiting (dopamine receptormediated) in conscious dogs (10). The 

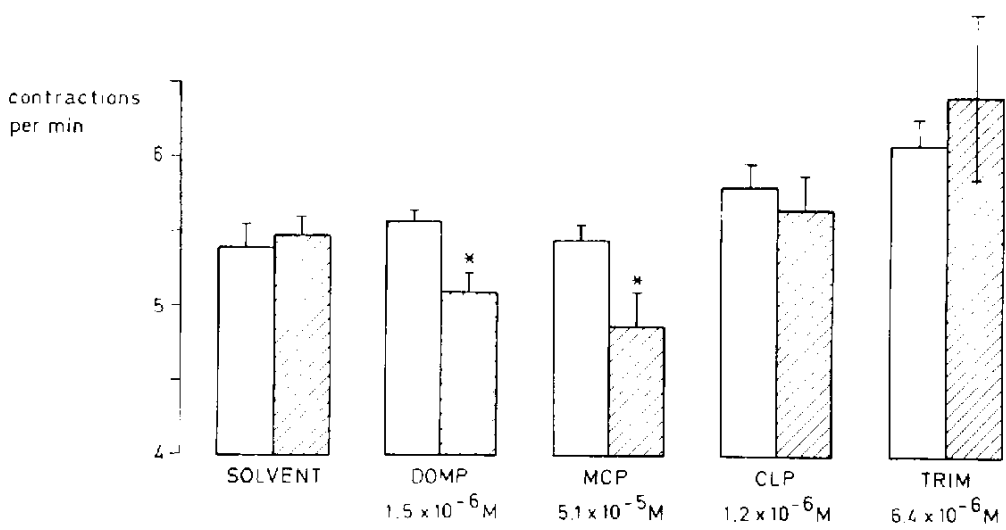

Fig. 6. Effects of domperidone (DOMP). metoclopramide (MCP), clebopride (CLP) and trimebutine (TRIM) at optimal concentrations on antral contractile frequency (in contractions per min) of the guinea pig stomach (open bars: initial values, hatched bars: values in the presence of the test compound, $\bar{m} \pm$ S.E.M., $n=5-9$. " significantly different from the initial value. $P<0.05$ ).

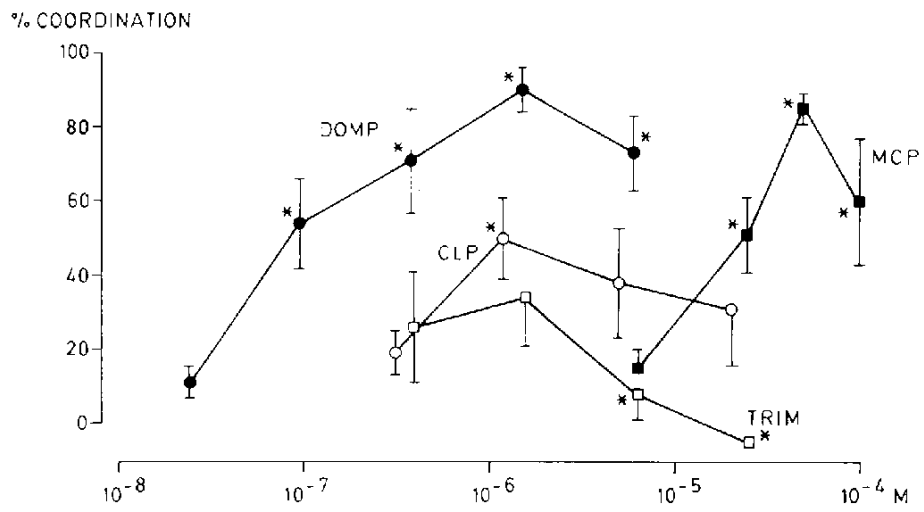

Fig. 7. Dose-response relation of the effects of domperidone (DOMP, O). clebopride (CLP. O). trimebutine (TRIM,,$j$ ) and metoclopramide (MCP. $\mathbf{D}$ ) on antroduodenal coordination in the guinea pig gastroduodenal preparation ( $\bar{m} \pm S . E . M ., n=5-9$. "significantly different from the initial value, $P<0.05$ ).

observation that domperidone reached its optimal effect after a longer incubation period than metoclopramide is in agreement with experimental data indicating a rapid but short-lasting action of the latter drug (11). Trimebutine was not tested against dopamineinduced gastric relaxations since the drug itself had a pronounced inhibitory effect on gastric tone; it induced a dose-dependent gastric relaxation. The EC50-value for this spasmolytic effect was similar to the EC5Ovalue for the inhibition of antroduodenal coordination by trimebutine. These concentrations as well as those at which trimebutine reduced the amolitude of the gastric contractions in our intact gastroduodenal preparation were similar to those reported for antral strips of the guinea pig $\left(10^{-6}-10^{-4} \mathrm{M}\right)$ $(12,13)$. This inhibitory action was not mediated via $\alpha$ - or $\beta$-adrenergic receptors (13) and as our results with domperidone indicate, also not via dopaminergic receptors. The insensitivity of the effect of trimebutine to naloxone indicates that in our model, opiate receptors are not involved in its action, in contrast to its effect on intestinal motility in the conscious dog (14).

Domperidone appeared most effective in 
stimulating rhythmic activity of the preparation. The concentrations needed to enhance antral contractile amplitude and to reduce frequency were 34 times below those needed for metoclopramide. Clebopride did not affect amplitude or frequency of the gastric waves, but it did enhance antroduodenal coordination at $1.2 \times 10^{-6} \mathrm{M}$. However. when the concentration was increased, this effect was lost. In contrast, both domperidone and metoclopramide enhanced antroduodenal coordination in a dosedependent manner, domperidone being 160 times as potent as metoclopramide. The broad effective dose range of domperidone and metoclopramide on antroduodenal coordination contrasts with the finding that their effects on gastric contractile activity were limited to a single concentration. indicating that the effects of the compounds on antroduodenal coordination are not caused by an effect on gastric contractile amplitude or frequency.

Clebopride is more effective than metoclopramide against dopamine-induced gastric relaxations, whereas metoclopramide is more effective on antroduodenal coordination. This paradox between two chemically closely related compounds remains to be solved. The relatively small effect of clebopride on antroduodenal coordination cannot be explained by its serotonergic blocking properties (6) since cisapride, a chemically-related compound (without dopamine-antagonistic properties) but also effective in antagonizing contractile responses to serotonin (15), does enhance antroduodenal coordination in this preparation in a dose-dependent manner $(9$. 16).

The findings with these four compounds may help to explain their effects in dyspepsia. Trimebutine is indicated in painful dyspepsia of spastic origin (17) in which the question remains whether either the upper gastrointestinal tract or the colon is causing the discomfort (18). In a double blind cross-over study. domperidone proved superior over trimebutine in functional dyspepsia (7). Domperidone and also metoclopramide have quite opposite effects to trimebutine in that they stimulate antroduodenal motility while moderately reducing gastric contractile fre- quency. A similar reduction of gastric frequency by domperidone was also observed in conscious dogs (11). Cooke and Stemper (19) showed that gastric emptying rate was optimal at a frequency of about 4 contractions per min in the dog. Therefore, a moderate reduction in contractile frequency may be important in man as well, in cases where gastric dysrhythmia (20) is the underlying cause of the dyspeptic complaint.

In conciusion: domperidone. clebopride. metoclopramide and trimebutine showed distinct effects on the gastroduodenal preparation of the guinea pig. Whereas trimebutine inhibited gastric tone, contractile amplitude and antroduodenal coordination as well, domperidone (low concentrations) and metoclopramide (high concentrations) enhanced amplitude and antroduodenal coordination but slightly reduced contractile frequency. Clebopride was relatively ineffective in enhancing gastric rhythmic activity but did antagonize the inhibitory action of dopamine on gastric tone as did domperidone and metoclopramide. These diverging actions may help explain the differences in patients' responsiveness to the treatment of digestive disorders such as dyspepsia or gastritis.

Acknowledgement: The authors are indebted to Drs. A.J. Reyntjens, W.J. Janssens and G.J.J.M. Vanderstichele for critical evaluation of the manuscript, to Mr. W.J.E. De Ridder for statistical analysis, to Mr. C.A.M. Van der Eycken for literature retrieval. Mr. J.M. Van Mierlo for preparing the illustrations. and Mrs. E.C.J. Wouters for secretarial assistance.

\section{References}

1 Brogden, R.N., Carmine, A.A., Heel, R.C., Speight, T.M. and Avery, G.S.: Domperidone. A review of its pharmacological activity. pharmacokinetics and therapeutic efficacy in the symptomatic treatment of chronic dyspepsia and as an antiemetic. Drugs 24, 360-400 (1982)

2 Van Nueten, J.M., Ennis, C., Helsen, L., Laduron, P.M. and Janssen, P.A.J.: Inhibition of dopamine receptors in the stomach: an explanation of the gastrokinetic properties of domperidone. Life Sci. 23, 453-458 (1978)

3 Laduron, P.M. and Leysen, J.E.: Domperidone, a specific in vitro dopamine antagonist, devoid of in vivo central dopaminergic activity. Biochem. Pharmacol. 28, 2161-2165 (1979)

4 Schuurkes, J.A.J. and Van Nueten, J.M.: 
Domperidone improves myogenically transmitted antroduodenal coordination by blocking dopaminergic receptor sites. Scand. J. Gastroenterol. 19, Supp. 96, 10†-110 (1984)

5 Kilbinger, H., Kruel, R., Pfeuffer-Friederich, I. and Wessler, 1.: The effects of metoclopramide on acetylcholine release and on smooth muscle response in the isolated guinea pig ileum. Naunyn Schmiedebergs Arch. Pharmacol. 319. 231-238 (1982)

6 Roberts, D.J.: The pharmacological basis of the therapeutic activity of clebopride and related substituted benzamides. Current Ther. Res. 31 , S1-S44 (1982)

7 Vuillez, J.P. and Laverdant, C.: Etude comparée du dompéridone et de la trimébutine dans le traitement de la dyspepsie fonctionnelle. Etude croisée en double insu. Lyon Médicale 253, 43-48 (1985)

8 Van Nueten, J.M. and Janssen, P.A.J.: Effect of domperidone on gastric relaxation caused by dopamine, secretin. 5-hydroxytryptamine, substance $P$, and adenosine triphosphate. In Gastrointestinal Motility, Edited by Christensen, J., p. 225-231. Raven Press. New York (1980)

9 Van Nueten, J.M., Leysen, J.E., Niemegeers, C.J.E. and Schuurkes, J.A.J.: Cholinergic vs anti-dopaminergic properties of prokinetic substituted benzamides with gastroin estinal prokinetic activity. Gastroenterology (Abstract) 88, 1623 (1985)

10 Niemegeers, C.J.E.: Antiemetic specificity of dopamine antagonists. Psychopharmacology (Berlin) 78, 210-213 (1982)

11 Shuto, K., Shiozaki, S., Kojima, T. and Tanaka, M.: Effects of KW-5338 (domperidone) on gastric motility. J. Pharmacobiodyn. 3, 715-719 (1980)

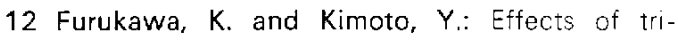
mebutine maleate (TM-906) on electrical and mechanical activities of smooth muscles of the guinea-pig stomach. Japan. J. Pharmacol. 35,
307-317 (1984)

13 Takenaga, H., Magaribuchi, T. and Tamaki, H.: Effects of trimebutine maleate (TM-906) on the spontaneous contraction of the isolated guinea pig stomach. Folia Pharmacol. Japon. 80 163168 (1982) (Abs, in English)

14 Fioramonti, J., Fargeas, M.J. and Bueno, L.: The involvement of opiate receptors in the effects of trimebutine on intestinal motility in the conscious dog. J. Pharm. Pharmacol. 36, 618621 (1984)

15 Schuurkes, J.A.J. and Van Nueten, J.M.: Evidence against a serotonergic mechanism for the motility stimulating properties of cisapride. Gastroenterology (Abstract) 88, 1577 (1985)

16 Van Nueten. J.M., Van Daele, P.G.H., Reyntjens, A.J., Janssen, P.A.J. and Schuurkes, J.A.J.: Gastrointestinal motility stimulating properties of cisapride, a non-antidopaminergic noncholinergic compound. In Gastrointestinal Motility. Edited by Roman. Cl., p. 513-520. MTP Press Ltd., Lancaster and Boston (1984)

17 Bonzi, G., Reguzzoni, G., Rocca, F, and Negri, L.: IL DA 3177, nuovo spasmolitico di sintesi, nella terapia degli stati spastici e discinetici dell'apparato digerente. Studio clinico controllato doppio cieco. Cl. Terap. 89, 187-196 (1979)

18 Lüttecke, K.: A three-part controlled study of trimebutine in the treatment of irritable colon syndrome. Curr. Med. Res. Opin. 6, 437-443 (1980)

19 Cooke, A.R. and Stemper, T.J.: The function of the antrum and pylorus in controlling emptying of liquid meals. In Proceedings of the Fifth International Symposium on Gastrointestinal Motility, Edited by Vantrappen, G., p. 278-283. Typoff-Press, Herentals (1976)

20 Chey, W.Y., You, C.H., Lee, K.Y. and Menguy, R.: Gastric dysrhythmia: clinical aspects. In Functional Disorders of the Digestive Tract, Edited by Chey, W.Y., p. 175-181, Raven Press, New York (1983) 